Supporting Information for:

\title{
2,2'-Bipyridyl as a Redox-Active Borylene Abstraction Agent
}

Siyuan Liu, a,b,c Marc-André Légaré, ${ }^{\mathrm{b}, c}$ Jens Seufert,, ${ }^{\mathrm{b}, \mathrm{c}}$ Dominic Prieschl,, ${ }^{\mathrm{b}, \mathrm{c}}$ Anna Rempel, ${ }^{\mathrm{b}, \mathrm{c}}$ Lukas Englert, ${ }^{\mathrm{b}, \mathrm{c}}$ Theresa Dellermann, ${ }^{\mathrm{b}, \mathrm{c}}$ Valerie Paprocki, ${ }^{\mathrm{b}, \mathrm{c}}$ Andreas Stoy, ${ }^{\mathrm{b}, \mathrm{c}}$ Holger Braunschweig ${ }^{*, b, c}$

a.Present address: School of Materials Science and Engineering, China University of Petroleum, Qingdao, Shandong, 266580, PR China

b. Institute for Inorganic Chemistry, Julius-Maximilians-Universität Würzburg, Am Hubland, 97074 Würzburg, Germany

c.Institute for Sustainable Chemistry \& Catalysis with Boron, Julius-Maximilians-Universität Würzburg, Am Hubland, 97074 Würzburg, Germany

*To whom correspondence should be addressed. E-mail: h.braunschweig@uni-wuerzburg.de 
NMR Spectra

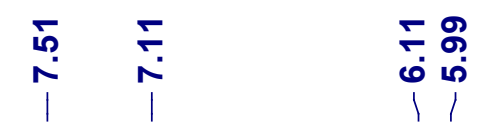

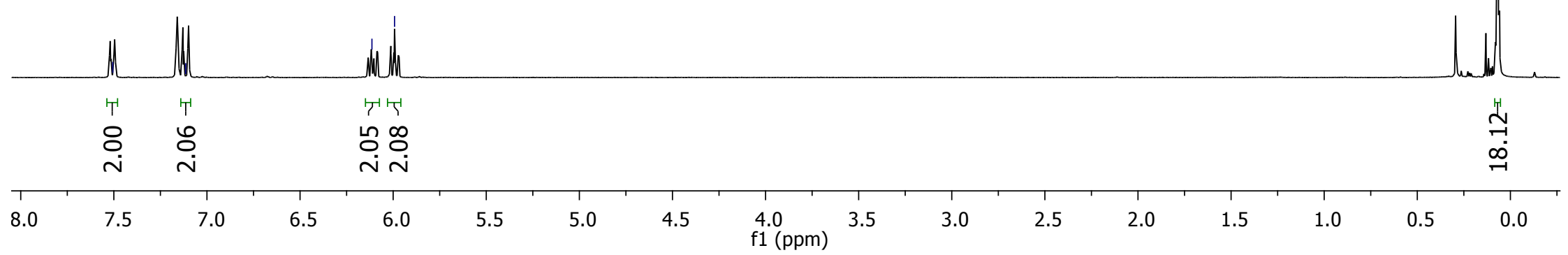

Fig. S1. ${ }^{1} \mathrm{H}$ NMR spectrum $\left(500 \mathrm{MHz}, \mathrm{C}_{6} \mathrm{D}_{6}\right.$, r.t.) of $\mathbf{3 a}$. 

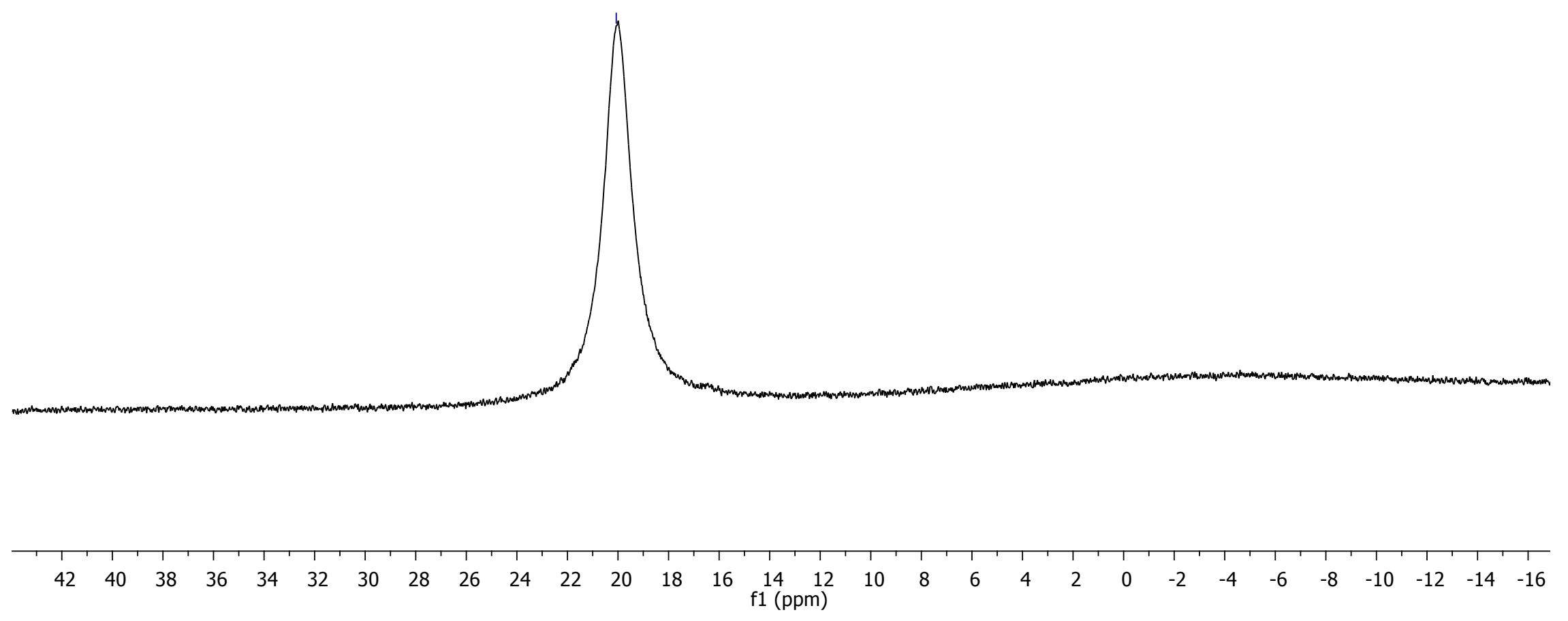

Fig. S2. ${ }^{11} B$ NMR spectrum ( $160 \mathrm{MHz}, \mathrm{C}_{6} \mathrm{D}_{6}$, r.t.) of $\mathbf{3 a}$. 


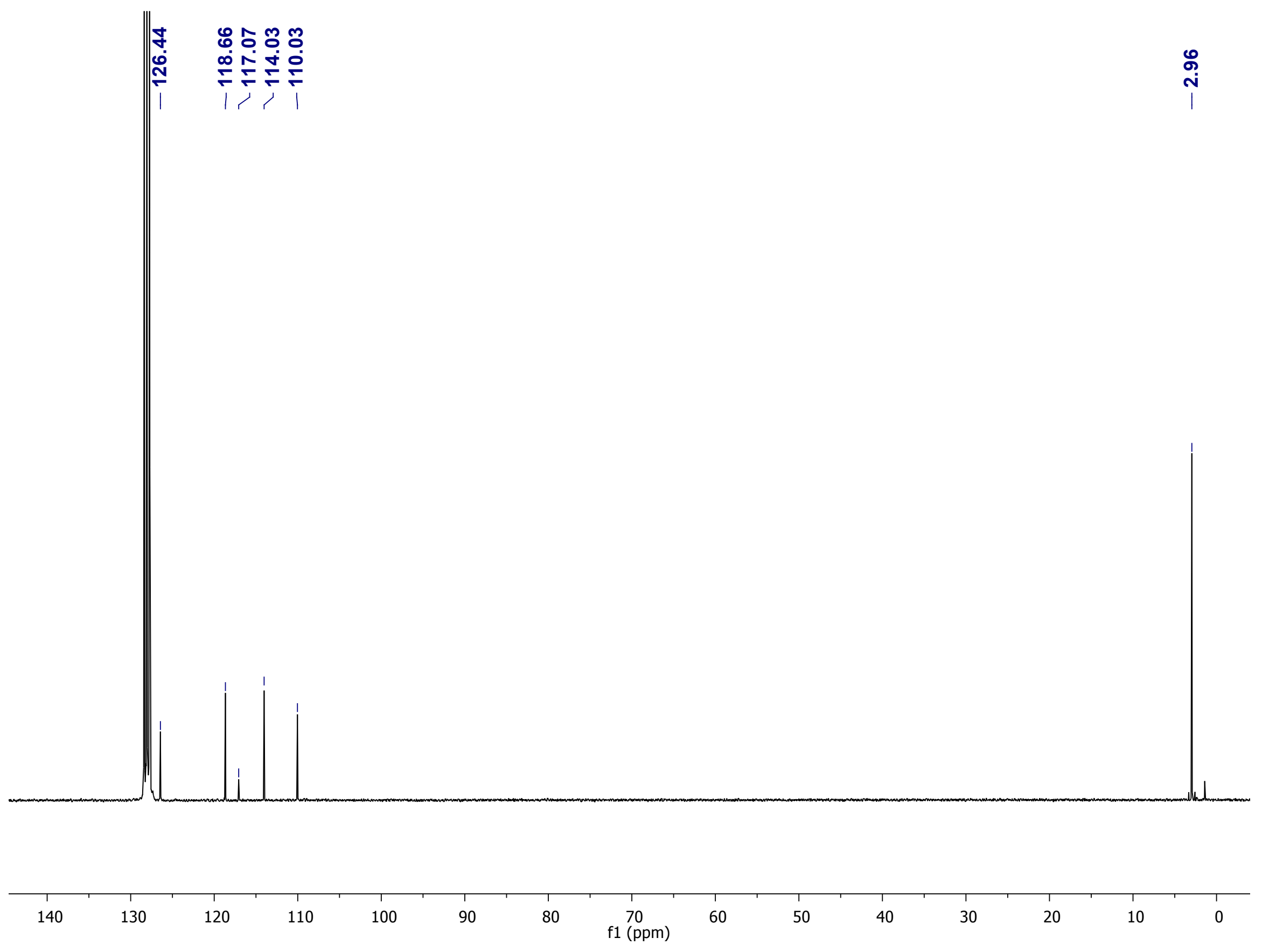

Fig. S3. ${ }^{13} \mathrm{C}\left\{{ }^{1} \mathrm{H}\right\}$ NMR spectrum (126 MHz, $\mathrm{C}_{6} \mathrm{D}_{6}$, r.t.) of $\mathbf{3 a}$. 
กิ ำ

NN

1)

ণิ ติ

i เ

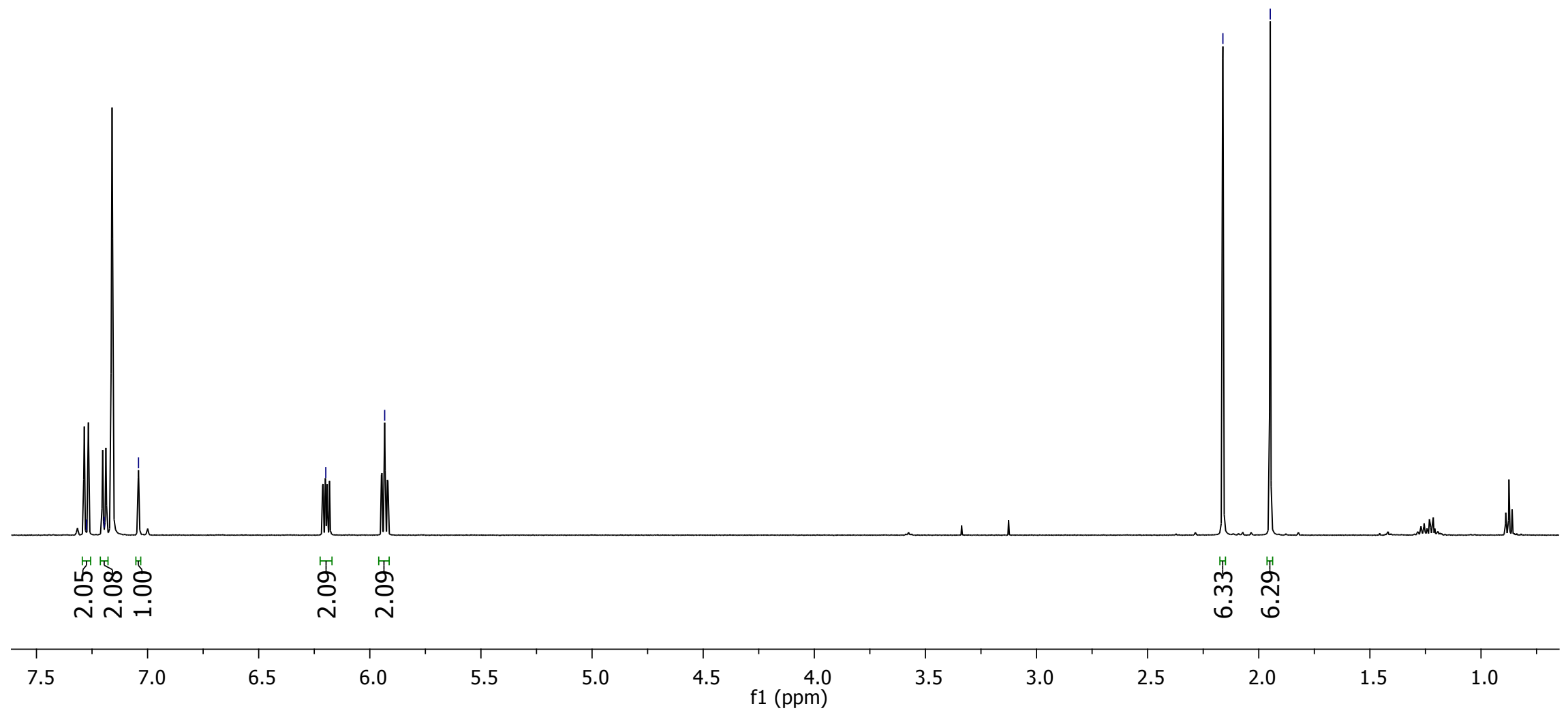

Fig. S4. ${ }^{1} \mathrm{H}$ NMR spectrum (500 MHz, $\mathrm{C}_{6} \mathrm{D}_{6}$, r.t.) of $\mathbf{3 b}$. 


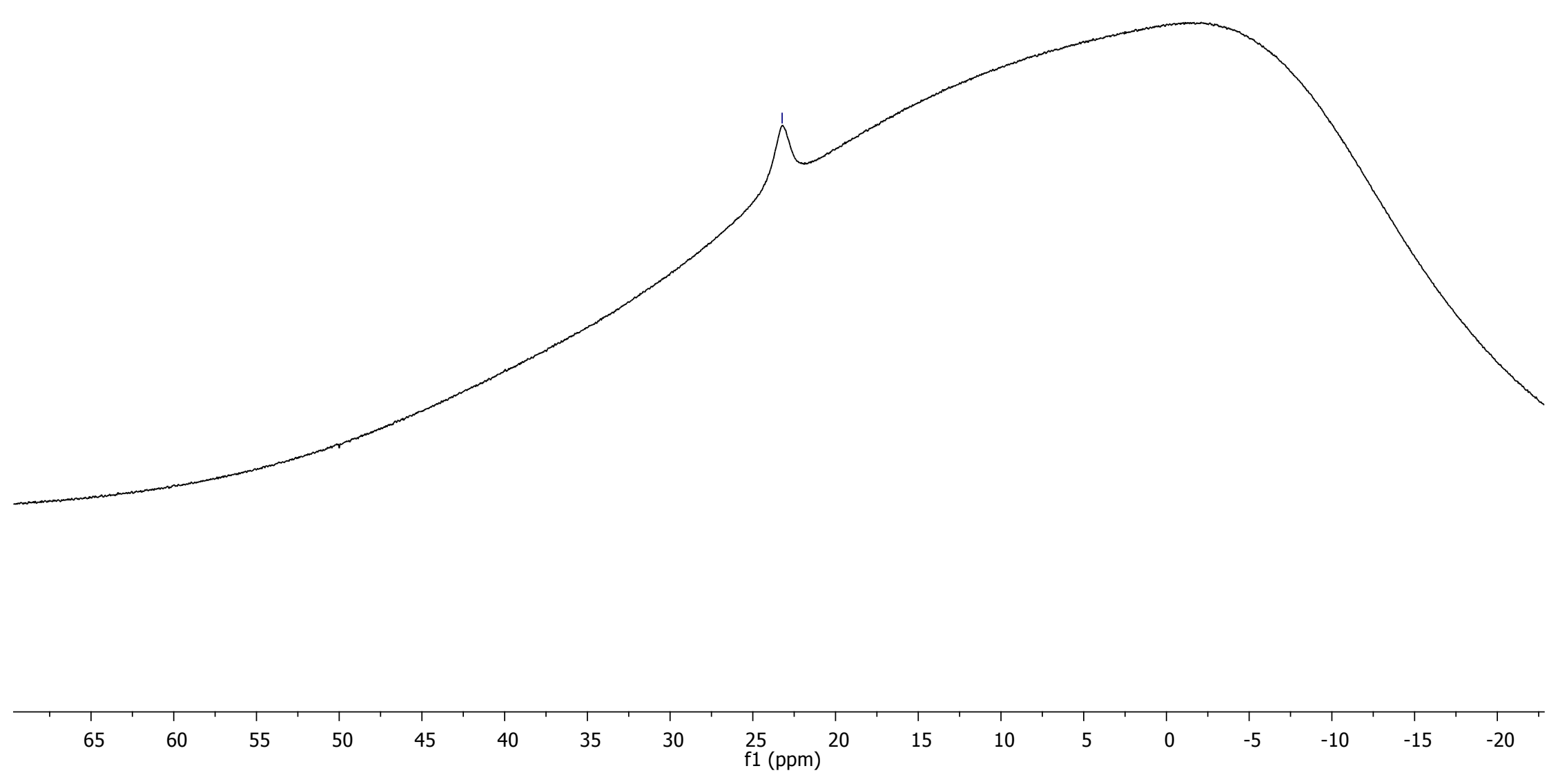

Fig. S5. ${ }^{11} \mathrm{~B}$ NMR spectrum (160 MHz, $\mathrm{C}_{6} \mathrm{D}_{6}$, r.t.) of $\mathbf{3 b}$. 

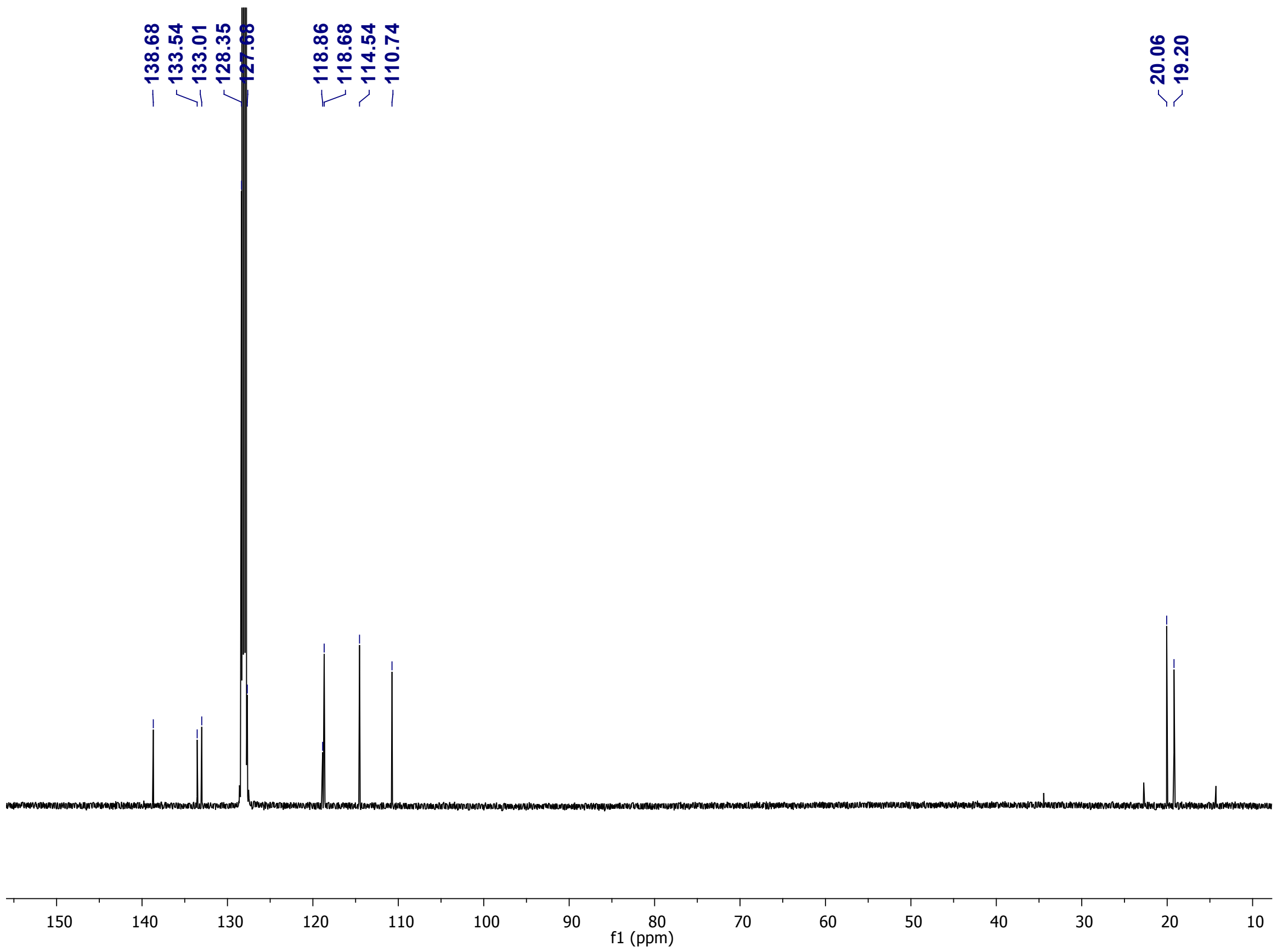

Fig. S6. ${ }^{13} \mathrm{C}\left\{{ }^{1} \mathrm{H}\right\}$ NMR spectrum (126 $\mathrm{MHz}, \mathrm{C}_{6} \mathrm{D}_{6}$, r.t.) of $\mathbf{3 b}$. 


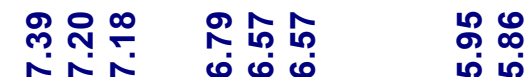

$\stackrel{0}{\stackrel{\circ}{i}} \stackrel{\infty}{i}$

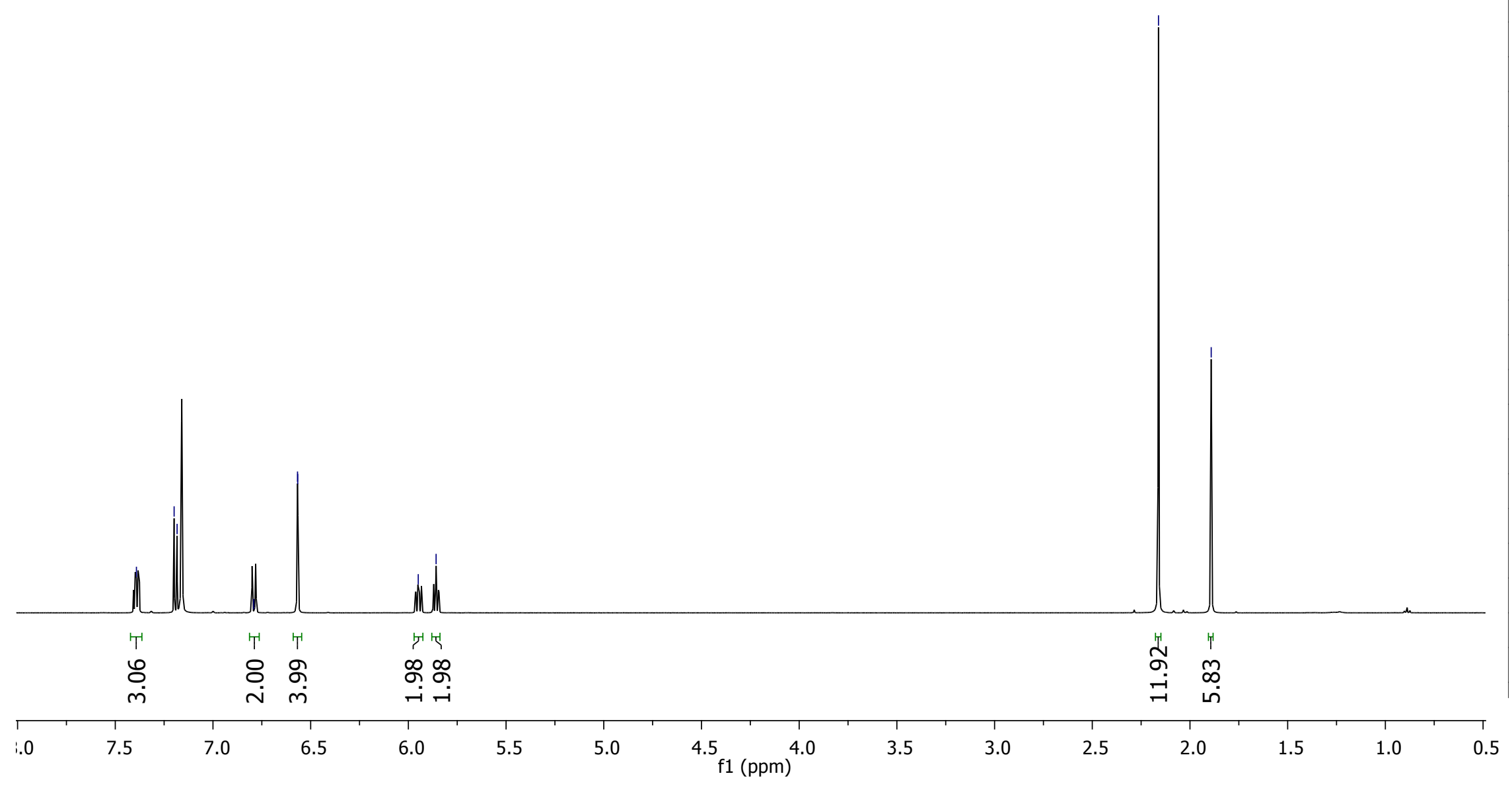

Fig. S7. ${ }^{1} \mathrm{H}$ NMR spectrum ( $500 \mathrm{MHz}, \mathrm{C}_{6} \mathrm{D}_{6}$, r.t.) of $\mathbf{3 c}$. 


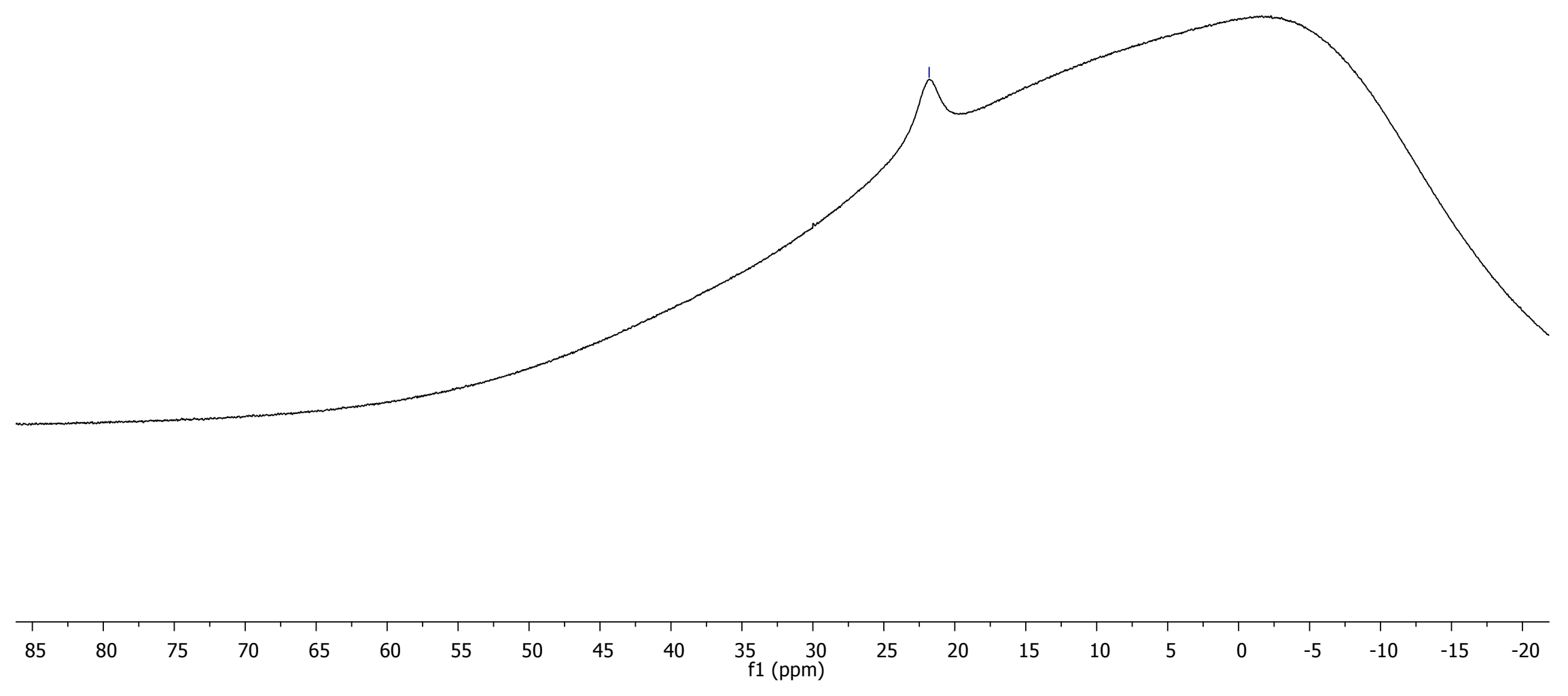

Fig. S8. ${ }^{11} \mathrm{~B} N M R$ spectrum $\left(128 \mathrm{MHz}, \mathrm{C}_{6} \mathrm{D}_{6}\right.$, r.t.) of $3 \mathrm{c}$. 


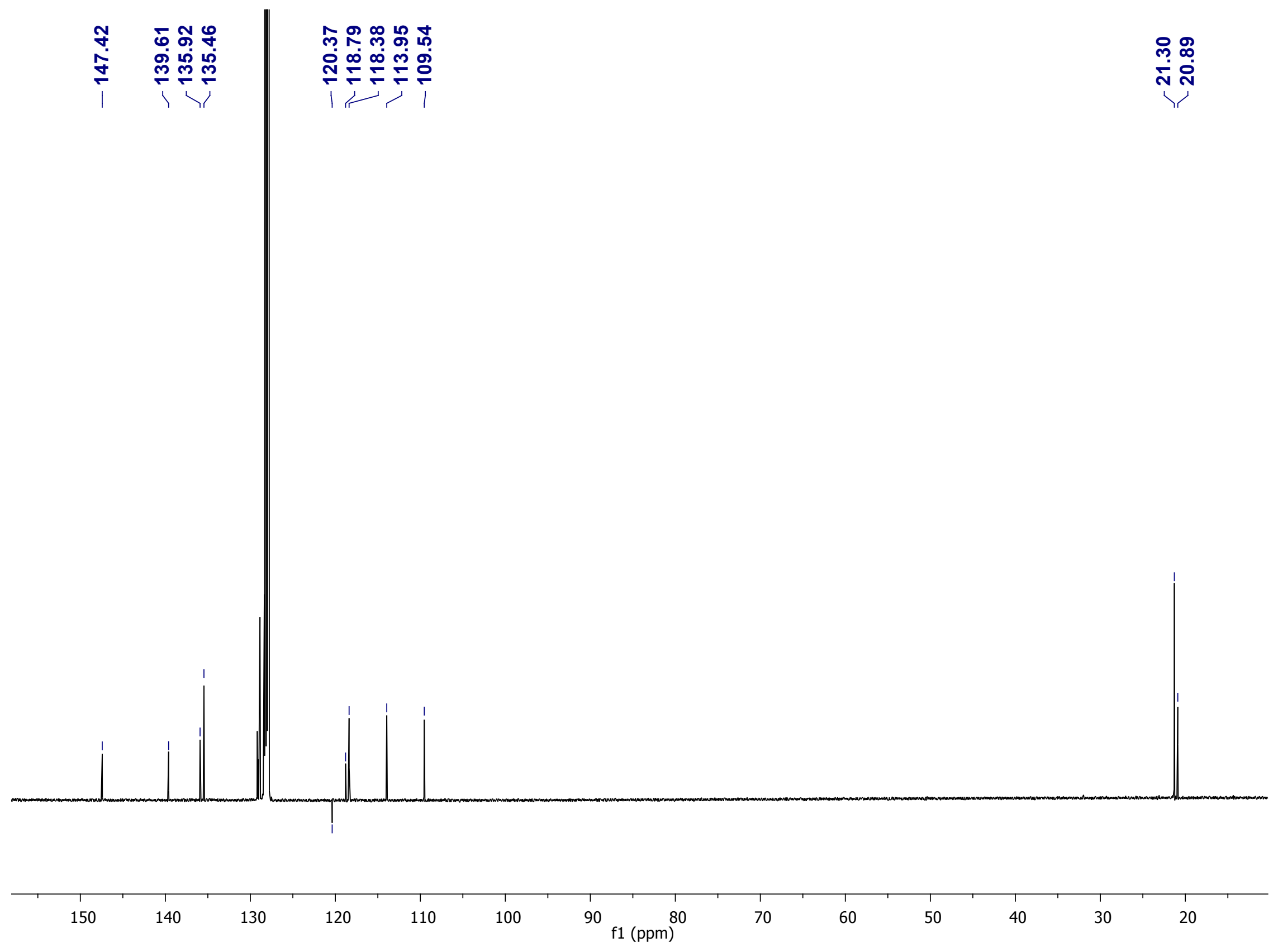

Fig. S9. ${ }^{13} \mathrm{C}\left\{{ }^{1} \mathrm{H}\right\}$ NMR spectrum (126 MHz, $\mathrm{C}_{6} \mathrm{D}_{6}$, r.t.) of $3 \mathrm{c}$. 


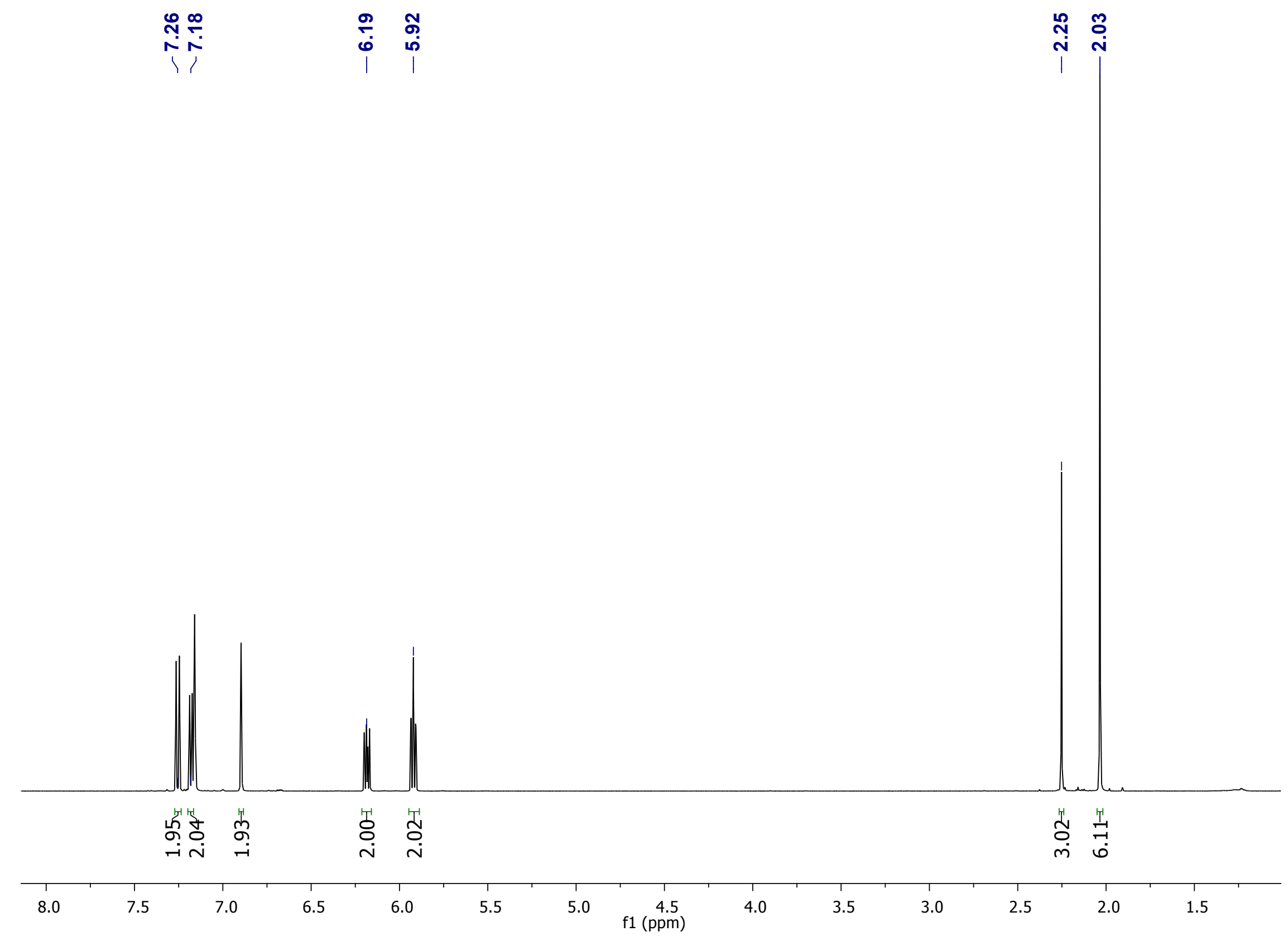

Fig. S10. ${ }^{1} \mathrm{H}$ NMR spectrum (500 MHz, $\mathrm{C}_{6} \mathrm{D}_{6}$, r.t.) of $\mathbf{3 d}$. 


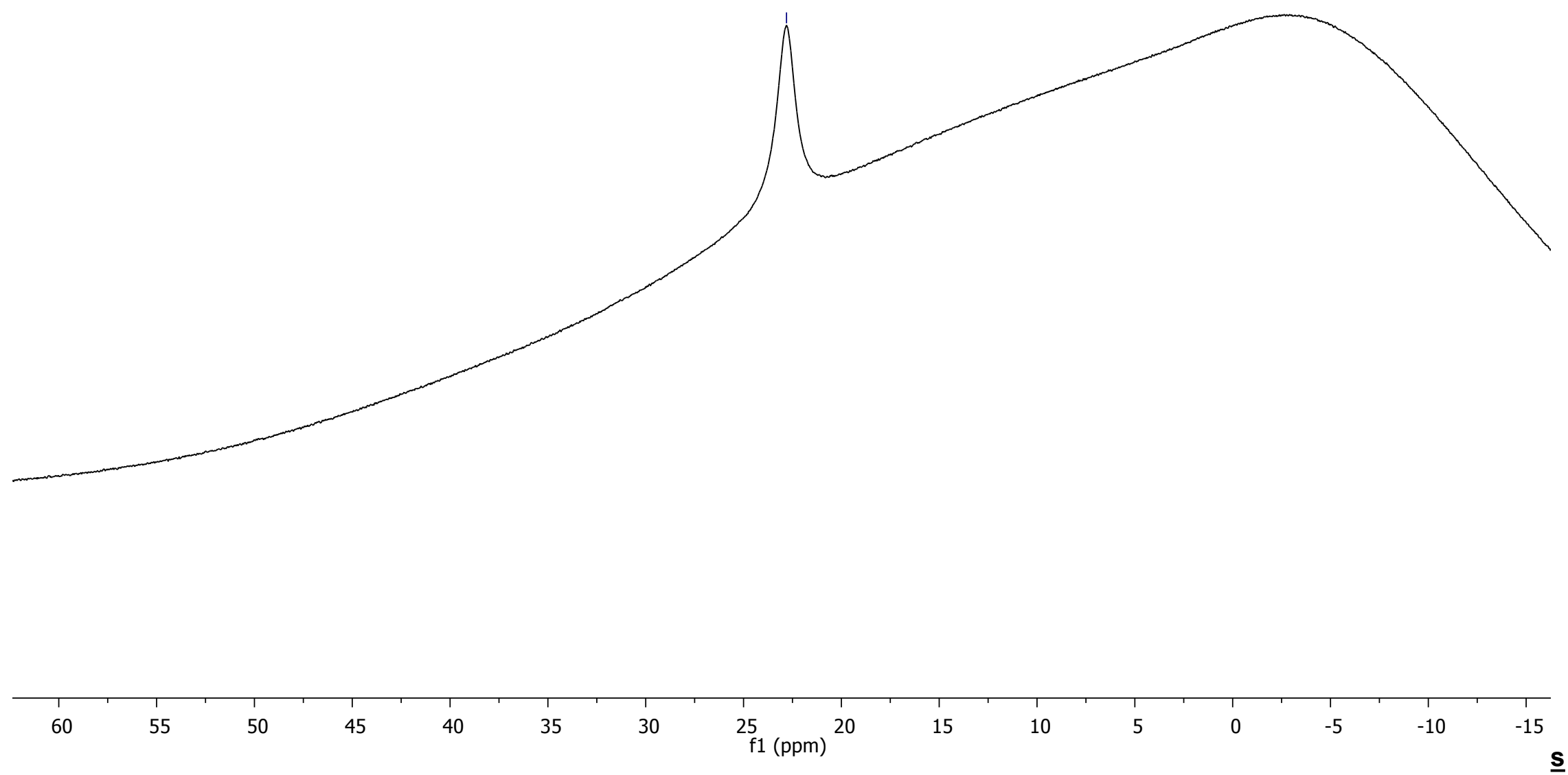

Fig. S11. ${ }^{11} \mathrm{~B}$ NMR spectrum (160 MHz, $\mathrm{C}_{6} \mathrm{D}_{6}$, r.t.) of $\mathbf{3 d}$. 


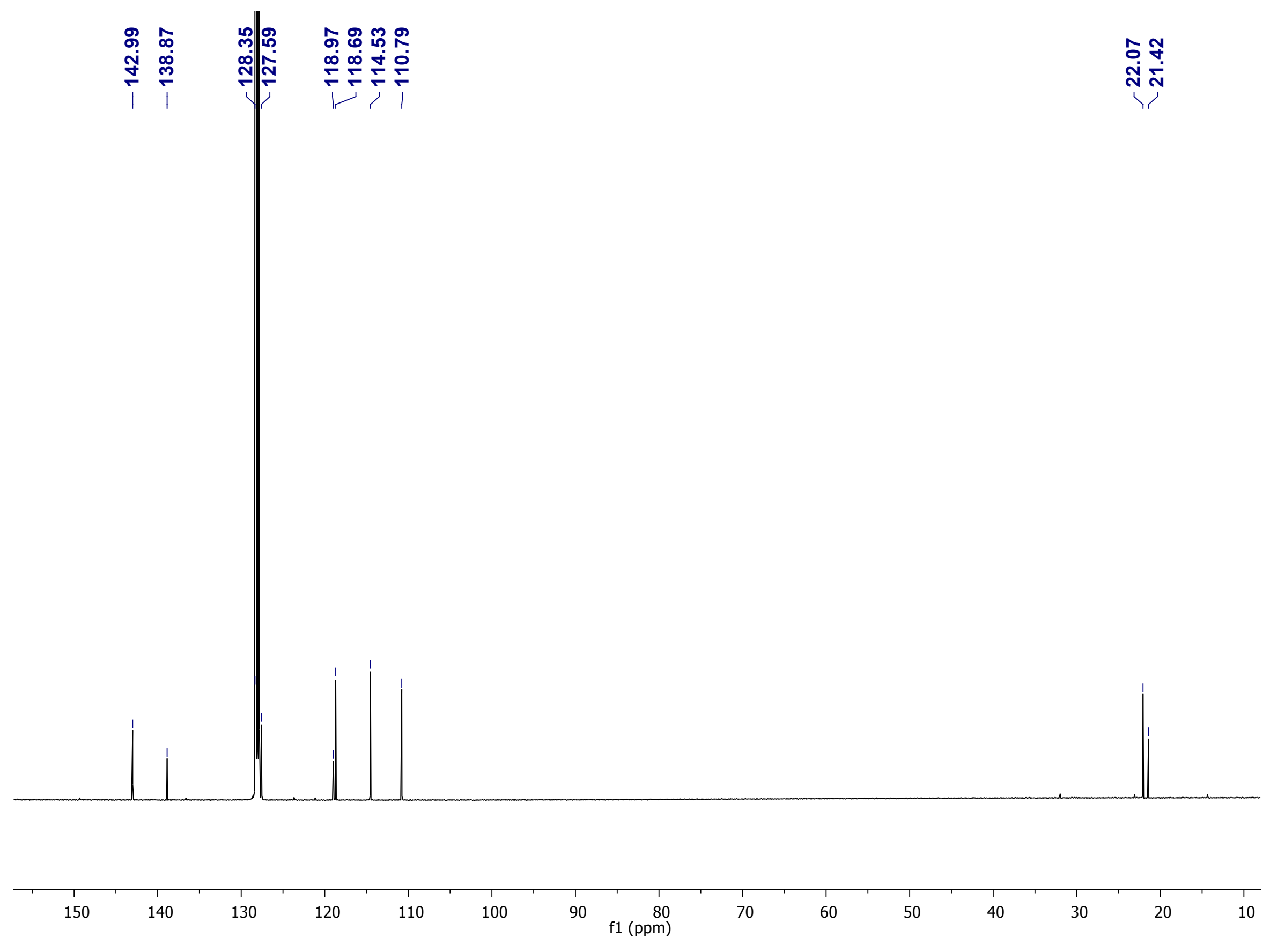

Fig. S12. ${ }^{13} \mathrm{C}\left\{{ }^{1} \mathrm{H}\right\}$ NMR spectrum (126 MHz, $\mathrm{C}_{6} \mathrm{D}_{6}$, r.t.) of $\mathbf{3 d}$. 


\begin{tabular}{|c|c|c|}
\hline 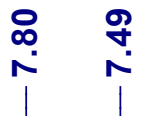 & 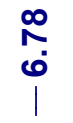 & $\begin{array}{l}\text { तु } \\
\text { i }\end{array}$ \\
\hline
\end{tabular}

$\stackrel{\text { no }}{\text { i }} \stackrel{i}{i}$

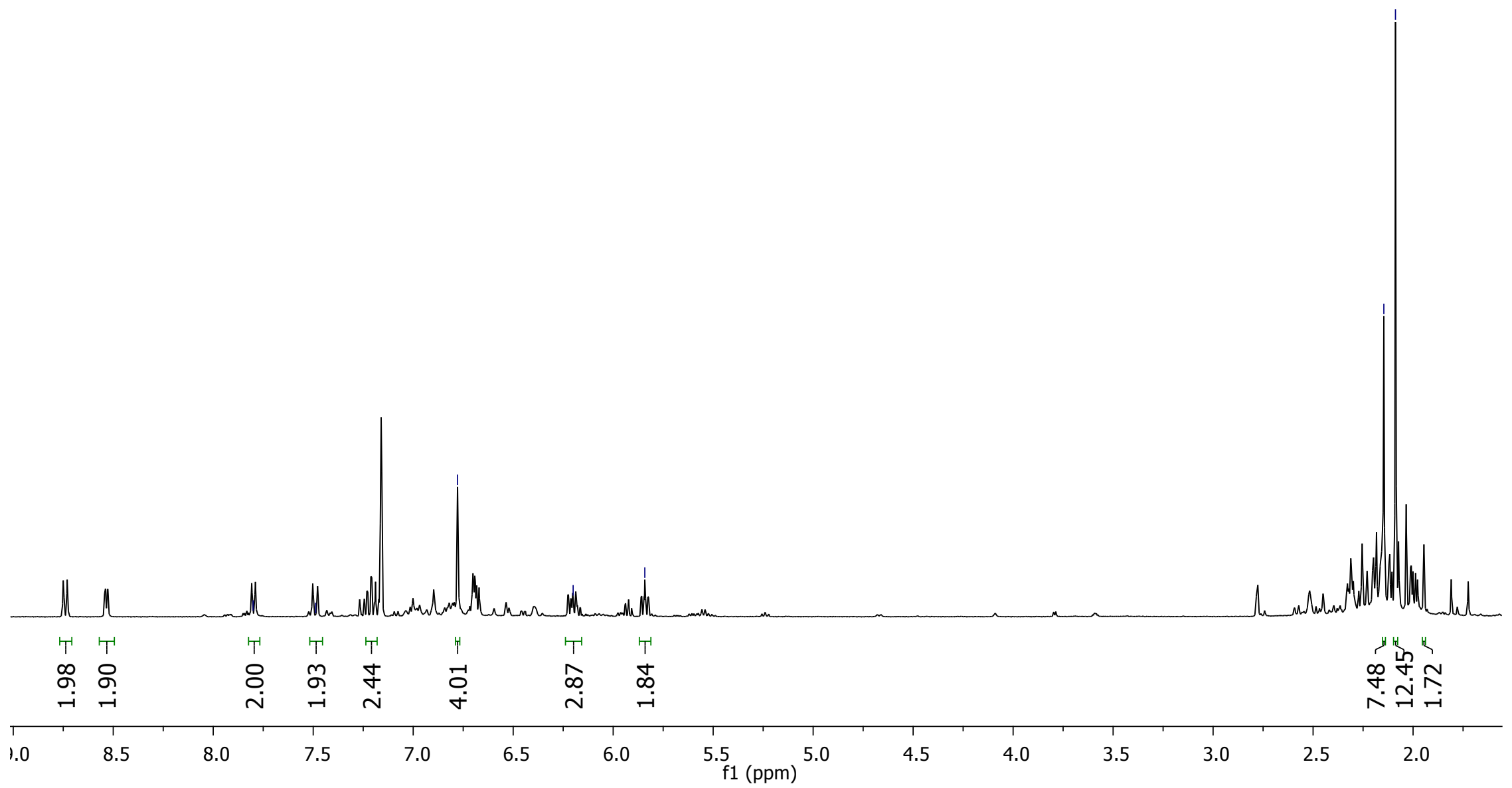

Fig. S13. ${ }^{1} \mathrm{H}$ NMR spectrum ( $400 \mathrm{MHz}, \mathrm{C}_{6} \mathrm{D}_{6}$, r.t.) of a crude reaction mixture of 8 and 1 in $\mathrm{C}_{6} \mathrm{D}_{6}$ (see above). The major product is 9 (peaks picked), in addition to free $\mathbf{1}, \mathbf{3 b}$ and thermal decomposition products of $\mathbf{8}$. 


$$
\stackrel{n}{i m}
$$


LIFD

RT: 0.00 - 3.59

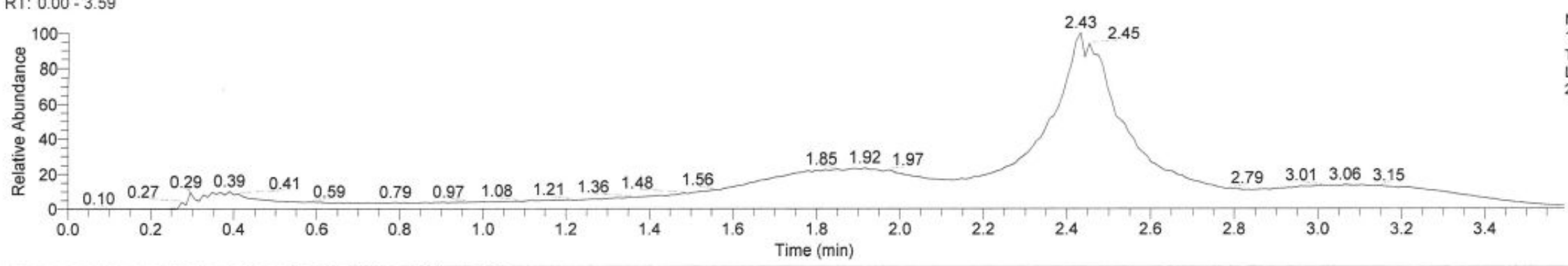

LE-383-2019-07-10-1 \#38-315 RT: 0.39-3.31 AV: 278 NL: 1.72E5

T: FTMS + p ESI Full ms2 1000.0000@hcd10.00 [150.0000-600.0000]

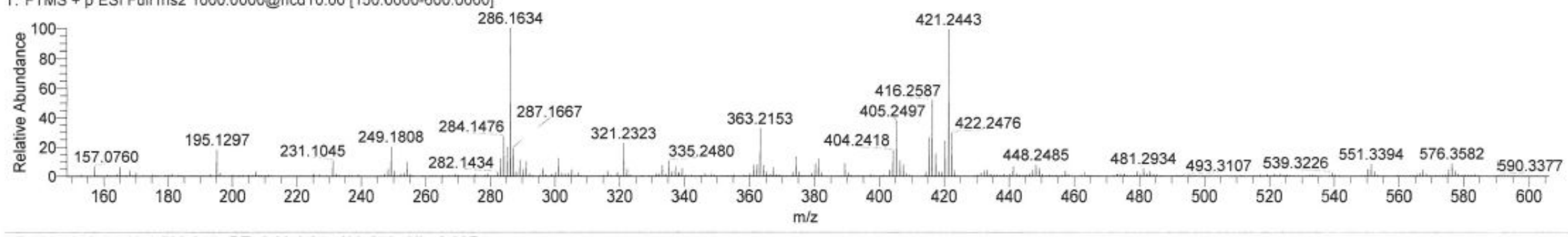

LE-383-2019-07-10-1 \#38-315 RT: 0.39-3.31 AV: 278 NL: 8.83E4

T: FTMS + p ESI Full ms2 1000.0000@hcd10.00 [150.0000-600.0000]

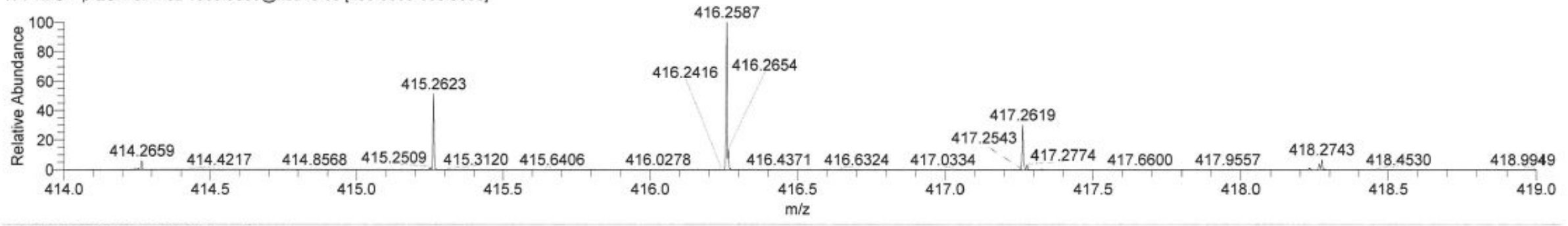

C28H30B2N2: C28 H3O B2 N2 pa Chrg 1

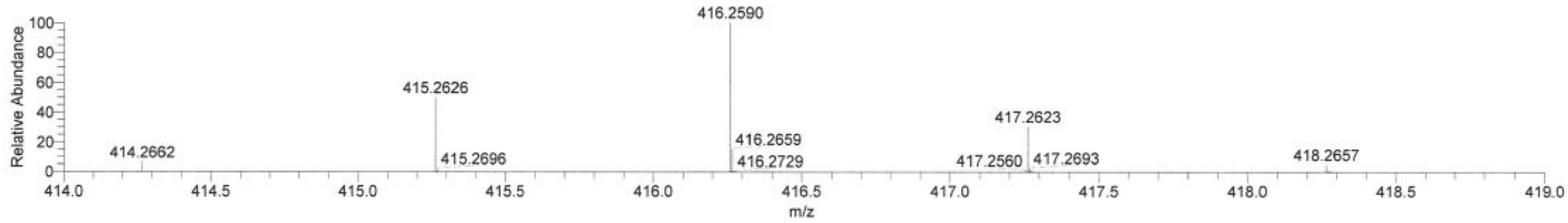

Fig. S15. HRMS spectrum of (LIFDI, toluene) of a crude reaction mixture of 8 and 1 in $\mathrm{C}_{6} \mathrm{D}_{6}$ (see above). Top: total ion count; second: mass distribution; third: zoom on the molecular ion peak of 9; bottom: simulated peak of $\mathbf{9}$. 
RT: $0.00 \cdot 3.59$

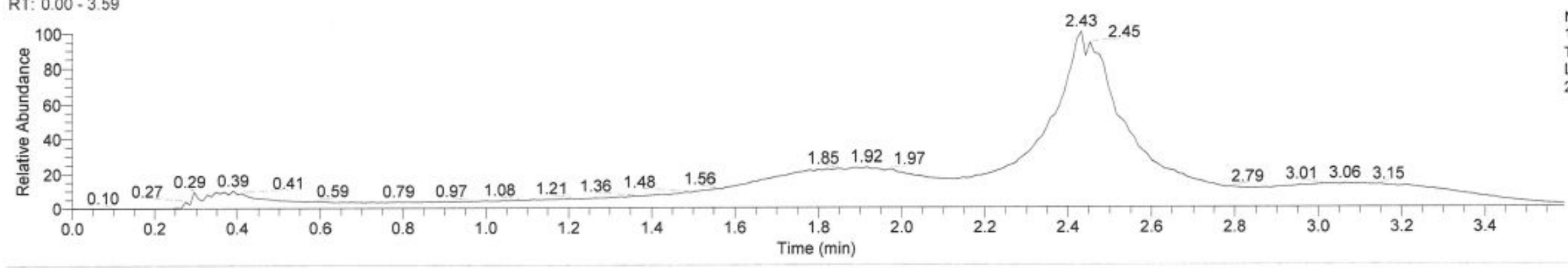

LE-383-2019-07-10-1 \#38-315 RT: 0.39-3.31 AV: 278 NL: 1.72 E5
T: FTMS + p ESI Full ms2 $1000.0000 @$ hed $10.00[150.0000-600.0000]$
286.1634

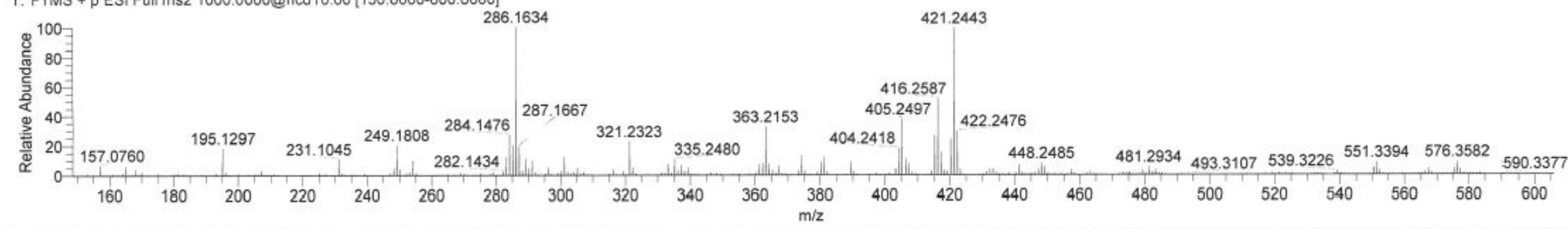

LE-383-2019-07-10-1 \#38-315 RT: 0.39-3.31 AV: 278 NL: $1.72 E 5$

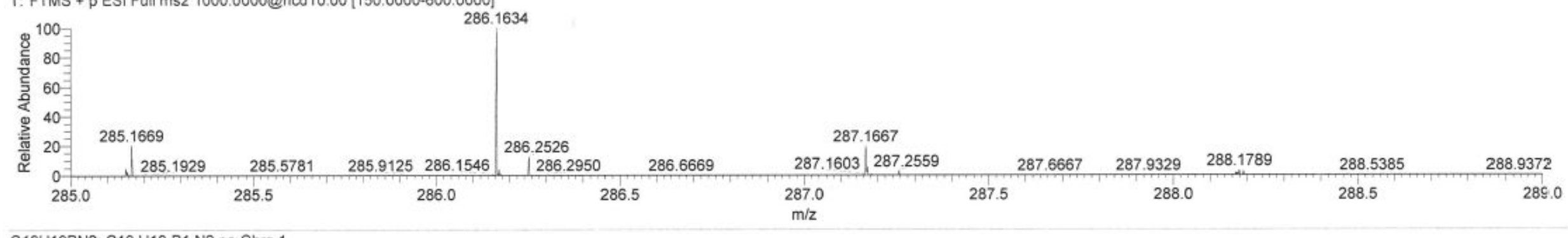

C19H19BN2: C19 H19 B1 N2 pa Chrg 1

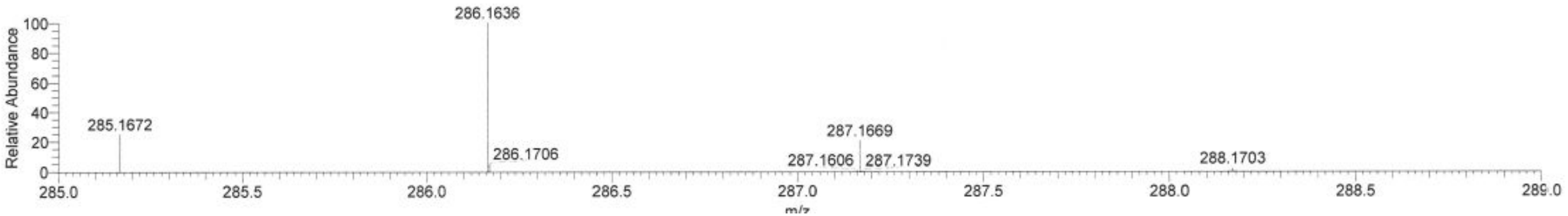

Fig. S16. HRMS spectrum of (LIFDI, toluene) of a crude reaction mixture of 8 and 1 in $\mathrm{C}_{6} \mathrm{D}_{6}$ (see above). Top: total ion count; second: mass distribution; third: zoom on the molecular ion peak of $\mathbf{3 b}$; bottom: simulated peak of $\mathbf{3 b}$. 


\section{Crystallographic Details}

The crystal data of compounds $\mathbf{3 b}$ and $\mathbf{3 d}$ were collected on a BRUKER D8 QUEST diffractometer with a CMOS area detector and multi-layer mirror monochromated $\mathrm{Mo}_{\mathrm{K \alpha}}$ radiation. The crystal data of compounds $\mathbf{3 c}$ and $\mathbf{9}$ were collected on a BRUKER SMART-APEX diffractometer with a $\mathrm{CCD}$ area detector and graphite monochromated $\mathrm{Mo}_{\mathrm{K} \alpha}$ radiation.

The structures were solved using intrinsic phasing method (SHELXT), ${ }^{1}$ refined with the SHELXL program $^{2}$ and expanded using Fourier techniques. All non-hydrogen atoms were refined anisotropically. Hydrogen atoms were included in structure factor calculations. All hydrogen atoms were assigned to idealised geometric positions.

Crystal data for $3 \mathbf{b}: \mathrm{C}_{20} \mathrm{H}_{21} \mathrm{BN}_{2}, M_{\mathrm{r}}=300.20$, red block, $0.159 \times 0.114 \times 0.06 \mathrm{~mm}^{3}$, monoclinic space group $P 2{ }_{1} / c, \quad a=13.136(6) \AA, \quad b=7.531(3) \AA, \quad c=17.643(7) \AA, \quad \beta=109.810(18)^{\circ}$, $V=1642.1(12) \AA^{3}, \quad Z=4, \quad \rho_{\text {calcd }}=1.214 \mathrm{~g}^{\cdot \mathrm{cm}^{-3}}, \quad \mu=0.070 \mathrm{~mm}^{-1}, \quad F(000)=640, \quad T=100(2) \mathrm{K}$, $R_{1}=0.1003, w R^{2}=0.1429,3350$ independent reflections $\left[2 \theta \leq 52.732^{\circ}\right]$ and 212 parameters.

Crystal data for $3 \mathrm{c}: \mathrm{C}_{34} \mathrm{H}_{33} \mathrm{BN}_{2}, M_{\mathrm{r}}=480.43$, red plate, $0.162 \times 0.092 \times 0.041 \mathrm{~mm}^{3}$, orthorhombic space group $P c a 2_{1}, a=31.889(5) \AA, b=10.2101(17) \AA, c=16.783(3) \AA, V=5464.4(15) \AA^{3}$, $Z=8, \quad \rho_{\text {calcd }}=1.168 \mathrm{~g} \cdot \mathrm{cm}^{-3}, \quad \mu=0.067 \mathrm{~mm}^{-1}, \quad F(000)=2048, \quad T=100(2) \mathrm{K}, \quad R_{1}=0.2046$, $w R^{2}=0.1388,9023$ independent reflections $\left[2 \theta \leq 52.042^{\circ}\right]$ and 679 parameters.

Crystal data for $3 \mathrm{~d}: \mathrm{C}_{19} \mathrm{H}_{19} \mathrm{BN}_{2}, M_{\mathrm{r}}=286.17$, red block, $0.32 \times 0.22 \times 0.15 \mathrm{~mm}^{3}$, monoclinic space group $P 2{ }_{1} / c, \quad a=13.4937(5) \AA, \quad b=29.6946(11) \AA, \quad c=7.9988(3) \AA, \quad \beta=96.369(2)^{\circ}$, $V=3185.3(2) \AA^{3}, \quad Z=8, \quad \rho_{\text {calcd }}=1.193 \mathrm{~g}^{\circ} \mathrm{cm}^{-3}, \quad \mu=0.069 \mathrm{~mm}^{-1}, \quad F(000)=1216, \quad T=296(2) \mathrm{K}$, $R_{1}=0.0474, w R^{2}=0.1254,6257$ independent reflections $\left[2 \theta \leq 52.042^{\circ}\right]$ and 403 parameters.

Crystal data for 9: $\mathrm{C}_{28} \mathrm{H}_{30} \mathrm{~B}_{2} \mathrm{~N}_{2}, M_{\mathrm{r}}=416.16$, red plate, $0.437 \times 0.251 \times 0.099 \mathrm{~mm}^{3}$, monoclinic space group $P 2_{1} / c, \quad a=8.669(4) \AA, \quad b=15.103(8) \AA, \quad c=17.642(9) \AA, \quad \beta=92.228(14)^{\circ}$,

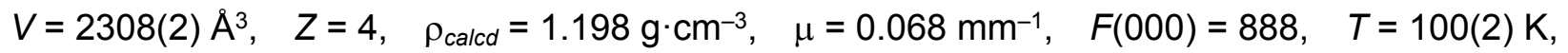
$R_{1}=0.0738, w R^{2}=0.1117,4553$ independent reflections $\left[2 \theta \leq 52.038^{\circ}\right]$ and 295 parameters.

Crystallographic data have been deposited with the Cambridge Crystallographic Data Center as supplementary publication no. CCDC 1986159-1986162. These data can be obtained free of charge from The Cambridge Crystallographic Data Centre via www.ccdc.cam.ac.uk/data_request/cif.

\section{$\underline{\text { References }}$}

1. G. Sheldrick, Acta Cryst., 2015, A71, 3-8.

2. G. Sheldrick, Acta Cryst., 2008, A64, 112-122. 